\title{
Analysis of Laboratory Surface Wave Data using the Scattering Transform for the Periodic Korteweg-de Vries
}

\author{
E. SEGRE, G. BOFFETTA and A. R. OSBORNE \\ Istituto di Fisica Generale, Università di Torino, Via Pietro Giuria 1, Torino 10125, Italy and Istituto di \\ Cosmogeofisica del C.N.R., corso Fiume 4, I-10133 Torino, Italy
}

and

M. PETTI

Dipartimento di Ingegneria Civile, Università Firenze, Via S. Marta 3, Firenze 50139, Italy

\begin{abstract}
We present a new approach which allows the analysis of nonlinear wave data within the framework of the periodic Korteweg-de Vries (KdV) equation. The method is based upon the periodic inverse scattering transform (IST) for $\mathrm{KdV}$, a nonlinear generalization of linear Fourier series, in which the signal is decomposed into a linear superposition of nonlinearly interacting hyperelliptic functions. The $\mathrm{KdV}$ is assessed as a model equation in a large number of physical problems, including $1+1$-dimensional nonlinear hydrodynamic wave propagation. We employ here IST to analyse laboratory surface wave data. Some physical implications are discussed; in particular we emphasize how the IST spectrum provides a much simpler interpretation of the wave motion than the linear Fourier transform, preserving the nonlinear mode amplitudes during the propagation of the waves.
\end{abstract}

\section{INTRODUCTION}

The Korteweg-de Vries equation is, as is well known, the prototypical soliton and integrable equation. The original discovery of multiple solitary wave interactions [1] led to the theoretical development of the mathematical method, known as inverse scattering transform (IST), which has since then been extended and applied to several other nonlinear equations and choices of boundary conditions [2,3]. We are here concerned with the propagation of water waves than are described by the KdV equation. Many aspects of this problem, particularly for motion dominated entirely by solitons, have already been studied $[2,4]$. Solitons, however, are phenomenologically understood as referring to a time asymptotic state in which individual pulses are well separated and singled out. This is the case in experimental studies, where often periodic or quasi-periodic boundary conditions are appropriate. We view the IST approach presented herein as a way to understand the dynamics of the nonlinear modes in complex systems of this type. The major goal of this paper is to show how this approach can be practically employed for the analysis of experimental data.

The paper is organized as follows. In Section 2 we recall the emergence of the $\mathrm{KdV}$ equation in the context of surface waves. Section 3 gives a very brief description of the underlying inverse scattering transform mathematical theory; Section 4 describes the characteristics of the wavetank facility and in Section 5 examples of recorded waves are presented, discussed and analysed. 


\section{KdV FOR SHALLOW WATER SURFACE WAVES}

The Korteweg-de Vries equation is derived in a multiscale expansion of the Euler equation for surface waves propagating over an horizontal bottom (see e.g. [2] for the details). The equation applies to the surface elevation $\eta(x, t)$ of $1 \mathrm{D}$, unidirectional long gravity waves in shallow water, in a regime where small but finite aspect ratio $\left(h_{0} / L\right)^{2}$ and relative height $|\eta| / h_{0}$ are of the samc order of magnitude. In the case we arc interested in, time series $\eta\left(x_{i}, t\right)$ recorded at fixed spatial locations $x_{i}$ are available; the IST method described below will then be applied to the time-like $\mathrm{KdV}$ equation (TKdV)

$$
\eta_{x}+\frac{1}{c_{0}} \eta_{t}-\frac{3}{2 h_{0} c_{0}} \eta \eta_{t}+\frac{h_{0}{ }^{2}}{6 c_{1]}{ }^{3}} \eta_{t t t}=0
$$

in which $h_{0}$ is the unperturbed water height, $c_{0}=\sqrt{ }\left(g h_{0}\right)$ and $g$ the acceleration of gravity. The exchange of space and time variables, with regard to what is generally held as the standard form of $\mathrm{KdV}$, is perfectly legitimate in the spirit of that multiscale expansion, and leads to the dimensional coefficients used in (1); it is consistent (at that perturbation order) with the substitution $\partial_{x} \rightarrow-\partial_{t} / c_{0}, \partial_{t} \rightarrow-c_{0} \partial_{x}$ into the traditional space-like KdV. TKdV solves a boundary value problem: given the temporal evolution $\eta\left(x_{0}, t\right)$ at some fixed spatial location $x_{0},(1)$ determines the wave motion over all space $\eta(x, t)$. In the present experiment we consider periodically generated waves, and hence periodic boundary conditions in $t$ are appropriate for the analysis which follows.

\section{SCATTERING TRANSFORM FOR THE PERIODIC KdV}

The scattering transform technique for soliton equations implies that spectral information can be recovered through the study of an associated eigenvalue problem. For the TKdV, the proper one is the one-dimensional Schrödinger equation

$$
\Psi_{t t}+\left[\lambda \eta\left(x_{0}, t\right)+E\right]^{\prime}=0
$$

where $\lambda=3 g / 2 h_{0}{ }^{3}$ and $E$ is the spectral parameter. We analyse the $M$-point equispaced periodic time series $\left\{\eta\left(x_{0}, t_{m}\right)\right\}, t_{m}=m T / M$, with $T$ the period. The problem is formulated in terms of the monodromy matrix $\alpha$, which maps values of $\left(\Psi, \Psi_{t}\right)$, solutions of (2), from $t$ to $t+T$ [5-8]. The matrix is computed under the piecewise-constant potential approximation [9] as

$$
\begin{aligned}
& \alpha(E)=\prod_{m=M}^{1} \alpha_{m}, \quad \text { where } \alpha_{m}=\left(\begin{array}{cc}
\cos \left(p_{m} \Delta t\right) & \frac{\sin \left(p_{m} \Delta t\right)}{p_{m}} \\
-p_{m} \sin \left(p_{m} \Delta t\right) & \cos \left(p_{m} \Delta t\right)
\end{array}\right) \\
& \text { and } p_{m}=\sqrt{ }\left[\lambda \eta\left(t_{m}\right)+E\right] \text {. }
\end{aligned}
$$

The spectrum of the signal is then defined from $\alpha$ as:

$$
\left.\begin{array}{rl}
\left\{E_{k}\right\} & =\{E \text { such that }|\operatorname{Tr}(\alpha(E))|=2\} \\
\left\{\mu_{j}\right\} & =\left\{E \text { such that } \alpha_{21}(E)=0\right\} \\
\left\{\sigma_{j}\right\} & =\left\{\operatorname{sgn}\left[\alpha_{11}(E)-\alpha_{22}(E)\right]_{E=\mu_{j}}\right\}
\end{array}\right\} \quad \text { main spectrum } \quad \text { auxiliary spectrum. }
$$

The discrete eigenvalues $E_{k}$ of the main spectrum are paired to define open bands $\left(E_{2 j}, E_{2 j+1}\right)$. When $E_{2 j}=E_{2 j+1}$ the band is called degenerate. The main spectrum comprises in general an infinite number of non-degenerate open bands, but their amplitude 
is rapidly decreasing as $E \rightarrow \infty$. Any smooth $\eta$ can be arbitrarily approximated by an $N$-band potential, e.g. by an IST solution with only $N$ non-degenerate bands, with $N$ large enough $[10,11]$. The main spectrum is invariant in space and time; this happens exactly because the potential $\eta$ in the Schrödinger equation satisfies the $K d V$, and it is the foundation of the IST approach. The auxiliary spectrum consists of hyperelliptic functions $\mu_{j}(x, t)$ which oscillate $j$ times during $T$ within the intervals $\left[E_{2 j}, E_{2 j+1}\right]$, according to the equations:

$$
\frac{\partial \mu_{j}}{\partial t}=\frac{2 \sigma_{j} \sqrt{ }\left[\prod_{k=1}^{2 N+1}\left(E_{k}-\mu_{j}\right)\right]}{\prod_{i \neq j}^{N}\left(\mu_{i}-\mu_{j}\right)}, \quad \frac{\partial \mu_{j}}{\partial x}=\left(4 \sum_{i \neq j}^{N} \mu_{i}-2 \sum_{k=1}^{2 N+1} E_{k}\right) \frac{\partial \mu_{j}}{\partial t} .
$$

Algorithms to efficiently compute the matrix, to precisely locate all the solutions in $E$ and to integrate $(5 a, b)$ are fully described in [9] and [12].

The solution to the periodic $\mathrm{KdV}$ equation may then be written ('first trace formula', [6]) as

$$
\lambda \eta(x, t)=-E_{1}+\sum_{j=1}^{N}\left[2 \mu_{j}(x, t)-E_{2 j}-E_{2 j+1}\right] .
$$

Just as ordinary Fourier analysis consists of the decomposition of a signal into a linear superposition of independent sine waves, IST views $\eta$ as a linear superposition of nonlinearly interacting, non-independent, hyperelliptic oscillation modes. The width of an open band here is the amplitude of a hyperelliptic oscillation mode, e.g. a 'single degree-of-freedom', 'spectral component' of $\mathrm{KdV}$, with amplitude and frequency

$$
a_{j}=\frac{E_{2 j+1}-E_{2 j}}{2 \lambda}, \quad v_{j}=\frac{j}{T} \text {. }
$$

In the absence of interactions among the nonlinear spectral components, that is in the limit $a_{j} \ll\left(E_{2 j+2}-E_{2 j-1}\right)$ for all $j$, or equivalently for infinitesimal $\eta(x, t)$, the $\mu_{j}(x, t)$ approach ordinary trigonometric functions. Equation (6) then reduces to an ordinary Fourier sereis [13].

In some cases one can qualitatively interpret the periodic KdV spectrum as separable into soliton and radiation components, although these are rigorously distinct entities only for infinite-line boundary conditions. This approach has also been used to perform a sort of nonlinear filtering of surface wave data in reference [14].

\section{DESCRIPTION OF THE WAVETANK}

We have performed several experiments of surface wave propagation in a wavetank facility at the University of Florence. The wavetank is a long and narrow channel of

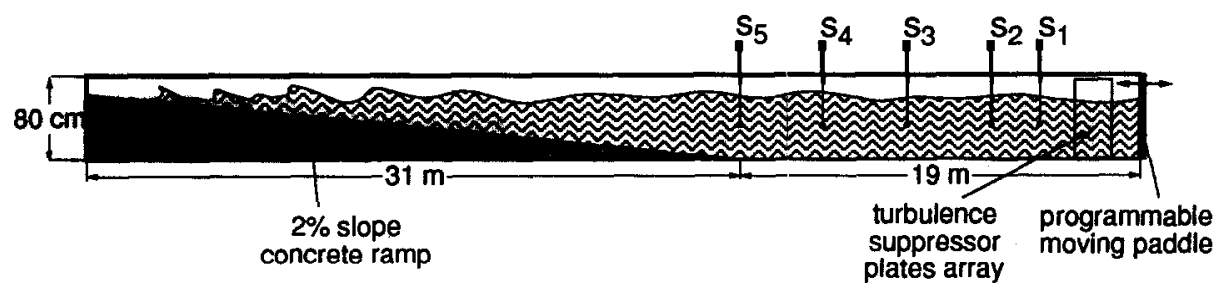

Fig. 1. Schematic representation of the wavetank. 
$50 \mathrm{~m} \times 0.8 \mathrm{~m} \times 0.8 \mathrm{~m}$ (Fig. 1), which can be filled with water up to a desired height. One end wall of the tank is an electronically controlled paddle that is moved for generation of surface waves. Oleodynamic circuitry and a position gauge for the feedback signal, enable exact placement and complete programmability of the movement. In front of the wavemaker, a fixed array of vertical plates aids in suppressing turbulence effects that may eventually be present.

At the opposite end of the channel a concrete rarap with a $2 \%$ slope acts as a wave absorber. This experimental arrangement is used to attain near unidirectional wave motion, dissipating the incoming waves by shoaling and breaking. The reflected wave amplitude is estimated to be smaller than $2 \%$ of the one of incoming waves. In this way and by choosing a consistent wavemaker drive we can attain surface wave motion in the tank in $\mathrm{KdV}$ regime.

Several wave height gauges (parallel wire resistance gauges) can be placed anywhere along the flume. In the present experiments we recorded the wave amplitudes $\eta\left(x_{i}, t\right)$ at $x_{1}=4.25, x_{2}=7.01, x_{3}=11.02, x_{4}=15.02$ and $x_{5}=19.01 \mathrm{~m}$ from the mean wavemaker position, in the centre of the channel. The output was sampled 20 times per second and converted to digital form with 12-bit resolution for computer acquisition. Both the wavemaker and the signal acquisition are completely controlled by an 80386-based computer running specific acquisition, preliminary analysis and control software.

Experimental uncertainties are estimated on the relevant measured quantities. The error analysis is shortly accounted into the appendix.

\section{WAVE TRAINS ANALYSIS}

We have generated waves of increasing nonlinearity in the channel by driving the wavemaker with a choice of smooth waveforms, including sine waves and low- $N$-band $\mathrm{KdV}$ potentials. Runs were conducted for $h_{0}$ equal to 30 and $49.8 \mathrm{~cm}$. and several wave amplitudes; in all cases considered the period of the wavemaker drive was chosen as $T=4 \mathrm{~s}$, well into the regime of long waves for these depths. Every run began from a still water condition, and we recorded time series for some minutes after the start of the wavemaker. A typical record is displayed in Fig. 2.

To perform the analysis, we selected subseries consisting of five wave periods starting $100 \mathrm{~s}$ after the initial time, simultaneously at each gauge. This was done in order to skip the initial transients, to exclude longer time scale irregularities due to residual reflected waves and to mediate over a few periods the slightly imperfect exact repetition of the generated waves. The subset was subsequently prefiltered with a linear low pass of $4.0 \mathrm{~Hz}$ to eliminate electronic noise, and then the Fourier and IST spectra were computed for each subseries. Figure 3(a) shows two periods of the subseries analysed, along with their decomposition into the 4 most energetic hyperelliptic modes (Fig. 3(b)). We can see that the nonlinear mode amplitudes are almost constant during the wave propagation; this means that the scattering transform decomposition reduces the complex wave evolution (Fig. 3(a)) to a few degree-of-freedom dynamics (Fig. 3(b)) according the KdV propagation.

In Fig. 4 we compare the linear Fourier spectrum (Fig. 4(a)) with the scattering transform spectrum (amplitudes of hyperelliptic modes $a_{j}$ as in (7)) (Fig. 4(b)), for the same 4 most energetic modes as they are seen at each station. For both methods, error analysis is performed; the less standard error propagation for IST is presented in the 


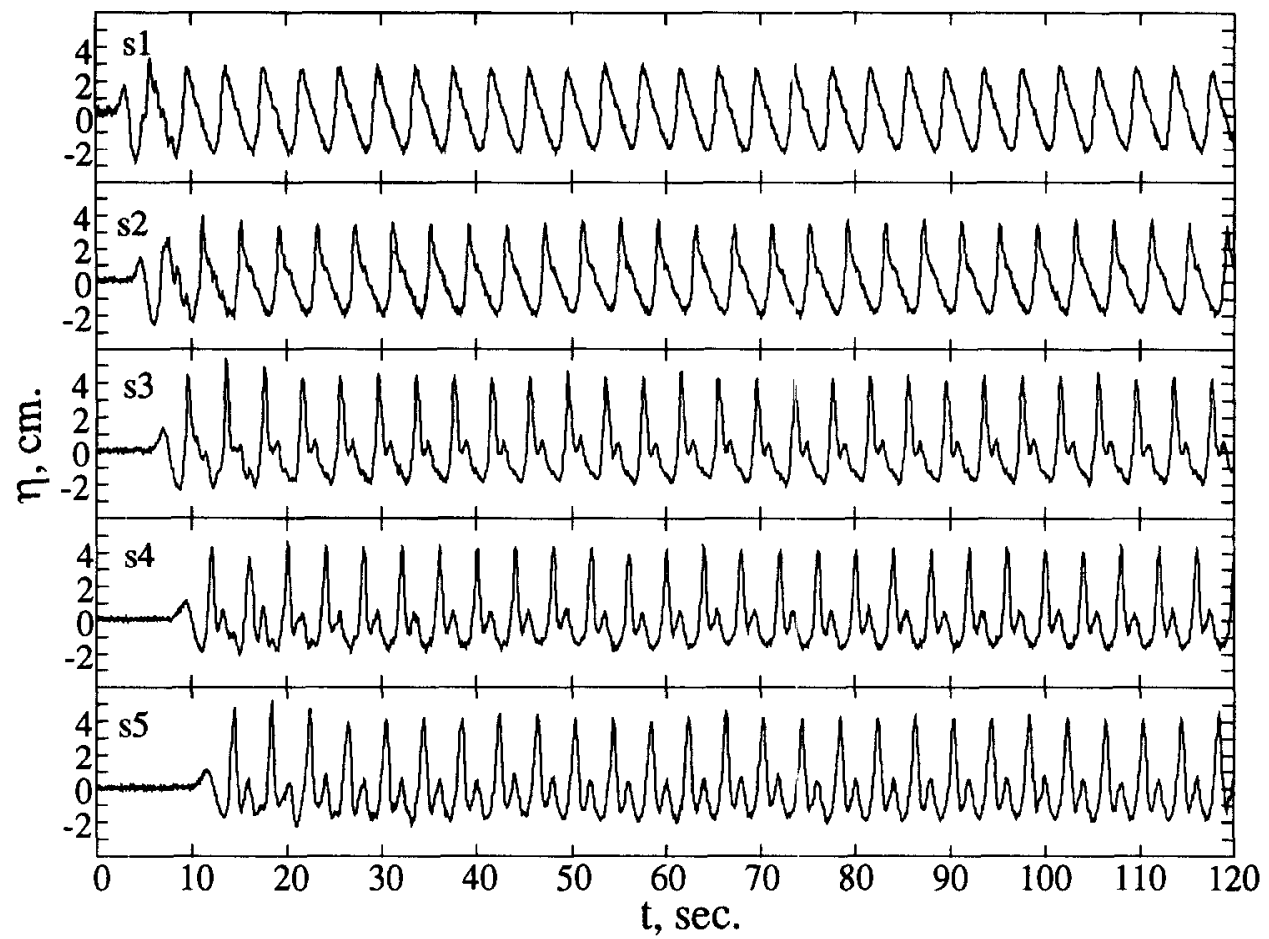

Fig. 2. Typical case raw gauge signals at the 5 probes, complete series, $h_{0}=30 \mathrm{~cm}$.

Appendix. Changes in the linear spectrum along the channel just tell us that the waves propagate according a nonlinear dynamics which couples the Fourier components in a complex way, so that the computation of the linear amplitude spectrum gives little physical insight; on the other hand, the almost constant scattering transform spectrum (at least within the experimental error bars) validates the TKdV equation as a good dynamical model.

Finally, as an additional test of the procedure, we numerically propagated signals from $x_{1}$ to $x_{5}$. Figure 5(a) compares $\eta\left(x_{5}, t\right)$ against $\eta\left(x_{1}, t\right)$ propagated as if the motion was linear, that is by antitransforming at $x_{5}$ the Fourier transform of $\eta\left(x_{1}, t\right)$; each spectral component is multiplied by a factor $\exp (i k(\omega) x)$, where $k(\omega)$ is found by numerically inverting the linear dispersion relation $\omega=\sqrt{ }\left[g k \tanh \left(k h_{0}\right)\right]$. Figure 5(b) instead compares $\eta\left(x_{5}\right)$ with the result of the numerical integration of $\eta\left(x_{1}, t\right)$ to $x_{5}$, according to a periodic TKdV scheme. Also for this direct test the superior performance the $\mathrm{KdV}$ dynamics is striking.

A proper non-dimensional parameter for the classification of the degree of nonlinearity of the wave field is the Ursell number $U r=3 \mathrm{HgT}^{2} / 4 h_{0}{ }^{2}$, for $H$ the peak-to-peak wave height. We have analysed with the above-described procedure six different cases, and tested the hypothesis of constant relevant $N$ spectral amplitudes, according both to Fourier and IST analysis. The goodness of fit is seen from the averaged reduced $\chi^{2}=$ $\sum_{j=1}^{N} \sum_{i=1}^{5}\left(a_{j}{ }^{(i)}-\overline{a_{j}}\right)^{2} / 4 N \sigma^{2}{ }_{j}^{(i)}$, where $a_{j}{ }^{(i)}$ is the amplitude of the $j$ th component at probe $i$, $\sigma^{2}{ }_{j}{ }^{(i)}$ its squared statistical error and $\overline{a_{j}}$ the mean value. We show in Fig. $6 \chi^{2}$ vs. the Ursell number. It is apparent how the hypothesis of constant Fourier amplitudes is completely rejectable as soon as the Ursell number increases, while still reasonable agreement can be assumed for the constancy of IST amplitudes. 

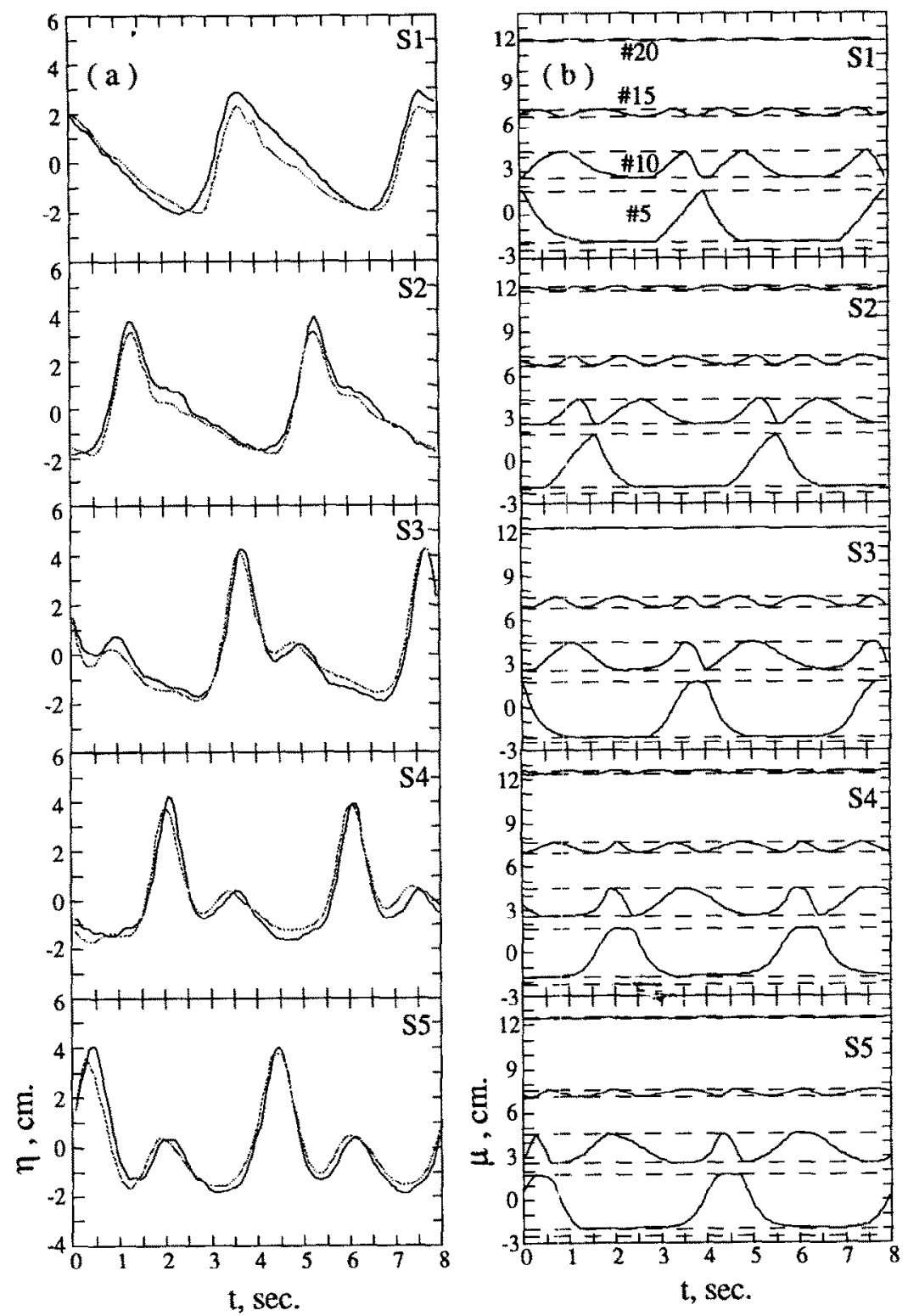

Fig. 3. IST decomposition of prefiltered data (a) original signals (continuous lines) and sum of the relevant modes (dotted lines); (b) individual hyperelliptic functions decomposition; for clarity just 2 of the 5 analysed periods are shown. 

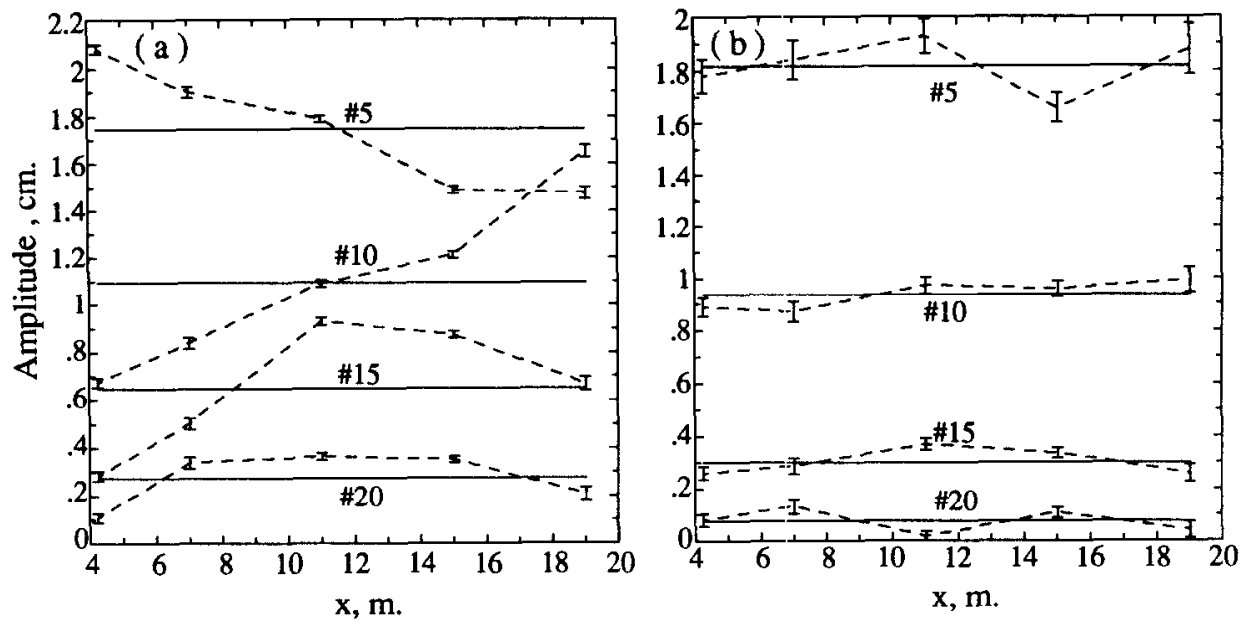

Fig. 4. Amplitudes of the four most energetic modes in the subseries along the channel: dashed lines: (a) linear Fourier components, (b) inverse scattering transform bands. Continuous lines: average amplitude of the individual modes at all probes.
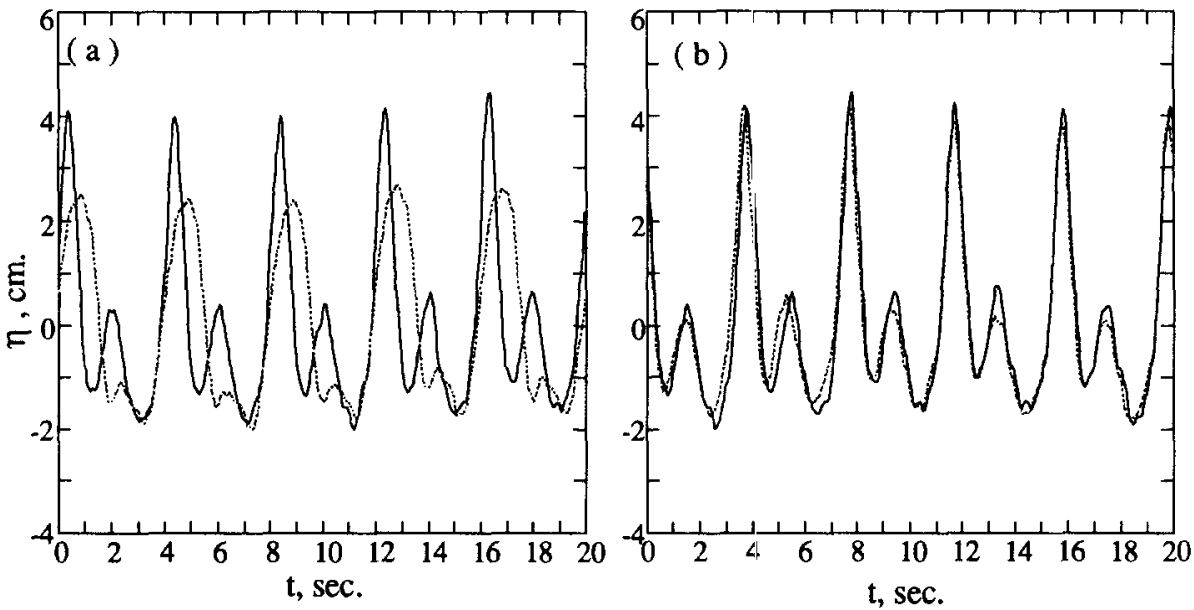

Fig. 5. Continuous line: measured signal at gauge S5; dotted line: numerical integration from probe S1 to S5 by (a) Fourier decomposition and linear evolution, (b) numerical integration of TKdV.

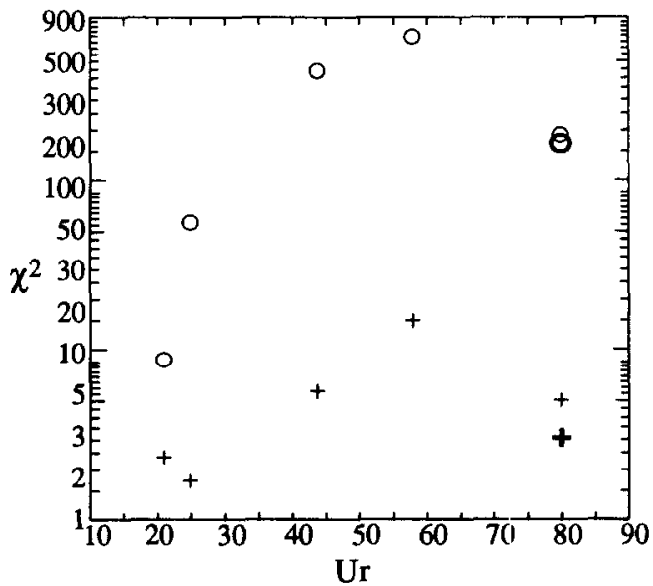

Fig. 6. Reduced values $\chi^{2}$ for the two hypothesis of $(O)$ Fourier and (+) IST constant mode amplitudes, for 6 experimental cases, versus Ursell number. Bold symbols refer to the case documented here. 


\section{CONCLUSIONS}

We have considered hydrodynamic waves in a regime where $\mathrm{KdV}$ theoretically holds, and analysed experimental data using the inverse scattering transform technique. Quantitative results and error analysis are achieved. Nonlinear Fourier amplitudes are found to be conserved during the propagation of the waves, while the linear Fourier has complex space-time dynamics. In this sense, considering wave motion in the context of a soliton equation, we have adopted a mathematical method which is closer to the structure of the nonlinear fluid dynamics of long wave motion. We therefore view the scattering transform approach as a promising technique for the nonlinear analysis of data.

\section{REFERENCES}

1. N. J. Zabusky and M. D. Kruskal, Interaction of 'solitons' in a collisionless plasma and the recurrence of initial states, Phys. Rev. Lett. 15, 240-243 (1965); C. S. Gardner, J. M. Greene, M. D. Kruskal and R. M. Miura, Method for solving the Korteweg-de Vries equation, Phys. Rev. Lett. 19, 1095-1097 (1967).

2. M. J. Ablowitz and P. A. Clarkson, Solitons, Nonlinear Evolution Equations and Inverse Scattering, Cambridge University Press, Cambridge (1991);

3. A. Degasperis, Nonlinear wave equations solvable by the spectral transform, in Nonlinear Topics in Ocean Physics. North-Holland, Amsterdam (1991).

4. J. L. Hammack and H. Segur, The Korteweg-de Vries equation and water waves, part 2. Comparison with experiments, J. Fluid Mech. 65, 289-314 (1974).

5. P. D. Lax, Periodic solutions of the KdV equation, Commun. Pare Appl. Math. 28, 141-188 (1975).

6. B. A. Dubrovin, V. B. Matveev and S. P. Novikov, Nonlinear equation of Korteweg-de Vries type, finite-zone linear operators, and Abelian varieties, Russian Math. Surv. 31(1), 59-146 (1976).

7. J. Garnett and E. Trubowitz, Gaps and bands of one dimensional periodic Schrödinger operators, Comment. Math. Helv. 59, 258-312 (1974).

8. H. Flaschka and D. W. McLaughlin, Canonically conjugate variables for KdV and Toda lattice under periodic boundary conditions, Proc. Theor. Phys. 55, 438 (1976).

9. A. R. Osborne and E. Segre, The numerical inverse scattering transform for the Korteweg-de Vries equation, Phys. Lett. A, 173, 131-142 (1993).

10. H. P. McKean and E. Trubowitz, Hill's operator and hyperelliptic function theory in the presence of infinitely many branch points, Commun. Pure Appl. Math. 29, 143-226 (1.976).

11. N. N. Meiman, The theory of one-dimensional Schrödinger operators with a periodic potential, J. Math. Phys. 18(4), 834-848 (1977).

12. A. R. Osborne and E. Segre, Numerical solutions of the Korteweg-de Vries equation using the periodic scattering transform $\mu$-representation, Physica $D, 44,575-604$ (1990).

13. A. R. Osborne and L. Bergamasco, The small-amplitude limit of the spectral transform for the periodic Korteweg-de Vries equation, Nuovo Cimento B 85, 229-243 (1985).

14. A. R. Osborne, E. Segre, G. Boffetta and L. Cavaleri, Soliton hasis stress in shallow-water ocean surface waves, Phys. Rev. Lett. 64(5), 592-595 (1991).

\section{APPENDIX: ERROR ANALYSIS}

At each probe we measure $h_{0}$ and the instantaneous wave displacement $\eta\left(t_{m}\right)$. We estimate a measurement error on $h_{0}$ to be $\sigma^{2}\left(h_{0}\right)= \pm 2 \mathrm{~mm} . \eta\left(t_{m}\right)$ is affected by errors due to slight nonlinearity of the gauges and to electronic noise. The first are evaluated from a three-point-fit of the calibration procedure readouts. Electronic noise is seen when looking at the zero-field signals, mostly as spurious small high frequency Fourier peaks. We consider $\eta\left(t_{i}\right)$ as affected by a gaussian error with a variance $\sigma^{2}(\eta)$ which is evaluated independently for each gauge, and is the order of $\pm 2 \mathrm{~mm}$.

An error on the $x$-position of the probes is estimated in $\pm 2 \mathrm{~cm}$, but is relevant only when considering integration (both linear or $\mathrm{KdV}$ ) from probe to probe. Other minor error sources that are less easily quantified, such as small dynamic displacements of the probes due to their flexibility, were ignored in this study. Quantities such as $\Delta t$ (derived from a digital timebase) are considered as unaffected by errors.

We report the basic error propagation formulae used for the treatment of our data. We have tried to apply the two techniques, Fourier and IST, in a consistent way, in order to compare the error bars of the two procedures when they are applied to periodic data. For the Fourier transform of the signal $\left\{\eta\left(t_{m}\right)\right\}$, defined as

$$
\hat{\eta}\left(\boldsymbol{v}_{j}\right)=\frac{1}{M} \sum_{m=1}^{M} \eta\left(t_{i}\right) \exp \left(-2 \pi i v_{j} t_{m}\right)
$$


where $v_{j}=j / M \Delta t, j=1, \ldots, M / 2$, the error on the amplitude $A\left(v_{j}\right)=\left|\hat{\eta}\left(v_{j}\right)\right|$ results in

$$
\sigma^{2}\left(A\left(v_{j}\right)\right)=\sum_{i=1}^{M}\left(\frac{\partial A\left(v_{j}\right)}{\partial \eta\left(t_{i}\right)}\right)^{2} \sigma^{2}\left(\eta\left(t_{m}\right)\right)==\frac{\sigma^{2}\left(\eta\left(t_{m}\right)\right)}{2 M} .
$$

The error analysis for IST is more involved, since the amplitudes are given implicitly. If each valuc $\eta\left(t_{m}\right)$ is varied of $\mathrm{d} \eta\left(t_{m}\right)$, we have to solve

$$
\operatorname{Tr}\left[\alpha\left(E, u\left(t_{i}\right)+\mathrm{d} u\left(t_{i}\right)\right)\right]_{E=E_{k}+\mathrm{d} E_{k}}= \pm 2
$$

instead of (6a) (note that the rescaled signal $u\left(t_{m}\right)=\lambda \eta\left(t_{m}\right)$ is used throughout the calculation). To the first order this implies

$$
\operatorname{Tr}\left[\alpha\left(E_{k}, u\left(t_{m}\right)\right)\right]+\frac{\partial \operatorname{Tr}\left[\alpha\left(E_{k}, u\left(t_{m}\right)\right)\right]}{\partial E} \mathbf{d} E_{k}+\sum_{m=1}^{M} \frac{\partial \operatorname{Tr}\left[\alpha\left(E_{k}, u\left(t_{m}\right)\right)\right]}{\partial u\left(t_{m}\right)} \mathbf{d} u\left(t_{m}\right)= \pm 2
$$

Exploiting $\mathrm{d} E_{k}$, we conclude that to the first order statistically

$$
\sigma^{2}\left(E_{k}\right)=\frac{\sum_{m=1}^{M}\left(\frac{\partial \operatorname{Tr}\left[\alpha\left(E_{k}, u\left(t_{m}\right)\right)\right]}{\partial u\left(t_{m}\right)}\right)^{2} \sigma^{2}\left(u\left(t_{m}\right)\right)}{\left(\frac{\partial \operatorname{Tr}\left[\alpha\left(E_{k}, u\left(t_{m}\right)\right)\right]}{\partial E}\right)^{2}}
$$

All terms in (A.5) are computable from the data, since

$$
\begin{gathered}
\frac{\partial \operatorname{Tr}\left[\alpha\left(E_{k}\right)\right]}{\partial u\left(t_{m}\right)}=\operatorname{Tr}\left[\left(\prod_{n=M}^{m+1} \alpha_{n}\right)\left(\begin{array}{cc}
-\frac{\sin \left(p_{m} \Delta t\right)}{2 p_{m}} \Delta t & \frac{\cos \left(p_{m} \Delta t\right)}{2 p_{m}^{2}} \Delta t-\frac{\sin \left(p_{m} \Delta t\right)}{2 p_{m}{ }^{2}} \\
\frac{\sin \left(p_{m} \Delta t\right)}{2 p_{m}}-\frac{\cos \left(p_{m} \Delta t\right)}{2} \Delta t & -\frac{\sin \left(p_{m} \Delta t\right)}{2 p_{m}} \Delta t
\end{array}\right)\left(\prod_{n=m-1}^{1} \alpha_{n}\right)\right. \\
\frac{\partial \operatorname{Tr}\left[\alpha\left(E_{k}, u\left(t_{m}\right)\right)\right]}{\partial E}=\sum_{m=1}^{M} \frac{\partial \operatorname{Tr}\left[\alpha\left(E_{k}, u\left(t_{m}\right)\right)\right]}{\partial u\left(t_{m}\right)} \\
\sigma^{2}\left(u\left(t_{m}\right)\right)=\lambda^{2} \sigma^{2}\left(\eta\left(t_{m}\right)\right)+\eta^{2}\left(t_{m}\right)\left(\frac{\partial \lambda}{\partial}\right)_{0}^{2} \sigma^{2}\left(h_{0}\right)
\end{gathered}
$$

Finally the error on $a_{j}$ is given by

$$
\sigma^{2}\left(a_{j}\right)=\frac{\sigma^{2}\left(E_{2 j}\right)+\sigma^{2}\left(E_{2 j+1}\right)}{\lambda^{2}}+\frac{\left(E_{2 j+1}-E_{2 j}\right)^{2}}{\lambda^{4}} \frac{81 \mathrm{~g}^{2}}{4 h_{0}^{4}} \sigma^{2}\left(h_{0}\right)
$$

where the errors $\sigma^{2}(E)$ are computed from (A.5). This latter result can be shown to tend to the one for the Fourier transform in the infinitesimal $\eta$ limit, by making use of the WKB approximation for (4). 